\title{
MANEJO PREOPERATORIO DE PACIENTES CON ENFERMEDADES RESPIRATORIAS CRÓNICAS*
}

\author{
Dr. Claudio Nazar J. ${ }^{1}$, Al. Roberto Coloma D. ${ }^{2}$, \\ Int. Maximiliano Zamora H. ${ }^{3}$, Dra. Isabel M. Leiva R. ${ }^{4}$
}

\author{
1 División de Anestesiología. \\ 2 Alumno de Medicina. \\ 3 Interno de Medicina. \\ 4 Departamento de Enfermedades Respiratorias, División de Medicina Interna. \\ Escuela de Medicina, Facultad de Medicina, Pontificia Universidad Católica de Chile. \\ Santiago, Chile.
}

\begin{abstract}
\section{Preoperative care of patients with chronic respiratory disorders}

Chronic respiratory disease is the $3^{\text {rd }}$ leading cause of death in Chile and its prevalence is increasing significantly in the world, so it is very common that we find patients with these pathologies undergoing elective surgical procedures. Perioperative complications are more frequent in these patients than in the general population, so it is essential adequate preoperative evaluation and management in them. Chronic obstructive pulmonary disease (COPD) is a major predictor of postoperative complications; therefore preoperative bronchodilators and inhaled corticosteroids are indicated in patients with this disease who will undergo elective surgery, particularly related to lung cancer. Likewise, COPD patients who will undergo coronary artery bypass graft surgery $(\mathrm{CABG})$ should receive oral or intramuscular corticosteroids preoperatively. Smoking is an independent risk factor for developing postoperative respiratory complications in thoracic surgery, so patients should stop this habit at least 4 weeks before elective surgery in order to significantly reduce such complications. A poorly controlled asthma is a specific risk factor for developing postoperative pulmonary complications, therefore these patients should be treated preoperatively with inhaled bronchodilators exclusive if they have mild asthma, bronchodilators associated with inhaled corticosteroids in the case of a moderate asthma, and inhaled bronchodilators associated to inhaled and oral corticosteroids, if they have severe asthma.

Key words: Surgery, care, preoperative, complications, postoperative, COPD, smoking, asthma.
\end{abstract}

\section{Resumen}

Las enfermedades respiratorias crónicas son la tercera causa de muerte en Chile y su prevalencia está aumentando significativamente en el mundo, por lo cual es muy frecuente que nos encontremos con pacientes con estas patologías sometiéndose a procedimientos quirúrgicos electivos. En estos casos las complicaciones

*Recibido el 14 de octubre de 2014 y aceptado para publicación el 12 de enero de 2015.

Los autores no refieren conflictos de interés.

Correspondencia: Dr. Claudio Nazar J. cenazar@med.puc.cl 
perioperatorias son más frecuentes que en la población general, por lo cual es indispensable una adecuada evaluación y manejo preoperatorio. La presencia de enfermedad pulmonar obstructiva crónica (EPOC) es un predictor mayor de complicaciones postoperatorias y deben indicarse broncodilatadores y corticoides inhalatorios preoperatorios en pacientes con esta patología que serán sometidos a cirugía electiva, especialmente en la relacionada a cáncer pulmonar. Así mismo, los pacientes con EPOC que se someterán a cirugía de revascularización miocárdica deben recibir además corticoides vía sistémica en el período preoperatorio. El tabaquismo es otro factor de riesgo independiente para desarrollar complicaciones respiratorias postoperatorias en cirugía torácica, por lo que los pacientes deberían suspender el consumo de tabaco por lo menos 4 semanas antes de la cirugía programada con el fin de disminuir significativamente dichas complicaciones. El asma bronquial mal controlada también es un factor de riesgo específico para desarrollar complicaciones pulmonares postoperatorias, por lo cual estos pacientes deben tratarse preoperatoriamente con broncodilatadores inhalatorios, asociados a corticoides inhalatorios u orales según la gravedad del asma.

Palabras clave: Cirugía, manejo preoperatorio, complicaciones postoperatorias, EPOC, tabaquismo, asma.

\section{Introducción}

Las enfermedades respiratorias corresponden a la tercera causa de muerte en Chile, solamente superadas por las enfermedades cardiovasculares y los tumores malignos ${ }^{1}$. Ha habido un aumento sostenido en la prevalencia de las enfermedades respiratorias crónicas en el mundo -como enfermedad pulmonar obstructiva crónica (EPOC), tabaquismo y asma bronquial- produciéndose un aumento del número de pacientes con estas patologías que serán eventualmente sometidos a un procedimiento quirúrgico. Según la Encuesta Nacional de Salud de 2009-2010, un $24,5 \%$ de los chilenos presenta síntomas respiratorios crónicos ${ }^{2}$, atribuibles a la contaminación ambiental y, principalmente, a que un $53,4 \%$ de los chilenos es o ha sido fumador alguna vez en su vida y un $40,6 \%$ fuma activamente en la actualidad ${ }^{3}$.

Las complicaciones pulmonares postoperatorias son frecuentes y aumentan la morbilidad, mortalidad y costos de salud, por lo cual requieren de un eficiente manejo preoperatorio. Johnson y cols. ${ }^{4}$, compararon los desenlaces de pacientes con y sin falla respiratoria -definida como ventilación mecánica postoperatoria mayor a $48 \mathrm{~h}$ o reintubación inesperada- como complicación postoperatoria en cirugías generales y vasculares. De los pacientes con falla respiratoria, un $26,5 \%$ falleció dentro de los primeros 30 días postoperatorios; $35,4 \%$ presentó una neumonía; $23,2 \%$ una sepsis y un $13,3 \%$ presentó un paro cardiorrespiratorio. En cambio, las tasas de cada uno de estos eventos fueron menores al $2 \%$ en los pacientes sin falla respiratoria.

El objetivo de esta publicación es revisar la evidencia actual del manejo preoperatorio de las tres patologías crónicas de origen respiratorio más frecuentes de encontrar en la población quirúrgica chilena: EPOC, tabaquismo y asma bronquial, buscando establecer las indicaciones preoperatorias que permitan a estos pacientes enfrentar el proce- dimiento quirúrgico en las mejores condiciones posibles.

\section{EPOC}

La iniciativa GOLD ${ }^{5}$ (Global Iniciative for Chronic Obstructive Lung Disease) ha definido EPOC como "una enfermedad común, prevenible y tratable, caracterizada por una persistente limitación al flujo aéreo que es normalmente progresiva y que se asocia con una respuesta inflamatoria, crónicamente exacerbada, de la vía aérea y los pulmones, frente a partículas y gases nocivos, contribuyendo las exacerbaciones y comorbilidades a la severidad global de los pacientes".

En Chile, el estudio PLATINO (Proyecto Latinoamericano de Investigación en Obstrucción Pulmonar $)^{6}$ demostró una prevalencia ajustada de EPOC de un $14,5 \%$, cifra que podría ser extrapolada a la población quirúrgica. La exacerbación del EPOC en el postoperatorio de pacientes sometidos a cirugía pulmonar - definida como la presencia de 3 de 5 de los siguientes criterios clínicos: aumento de la disnea, esputo purulento, volumen de secreción bronquial $>10 \mathrm{ml} * \mathrm{~kg}^{-1}$, fiebre sin causa aparente y sibilancias- aumenta las tasas de complicaciones respiratorias generales, estadía hospitalaria y mortalidad $^{7}$. Una revisión sistemática realizada por el American College of Physicians el $2006^{8}$, encontró que en 13 de 15 estudios reportaron que la presencia de EPOC era un predictor de complicaciones pulmonares postoperatorias, haciendo necesario un adecuado manejo preoperatorio de los pacientes que padecen EPOC, de manera de disminuir eventuales complicaciones postoperatorias.

Arozullah y cols. ${ }^{9,10}$, publicaron el 2000 y 2001 un estudio de cohorte (18.1109 y 31.6071 pacientes, respectivamente), determinando un índice de riesgo para el desarrollo de falla respiratoria o neumonía postoperatoria en cirugías no torácicas. En ambos 
estudios EPOC resultó ser un predictor mayor de complicaciones respiratorias postoperatorias [falla respiratoria: Riesgo Relativo (RR) 1,81 (IC 1,661,98); neumonía RR 1,72 (IC 1,55-1,91)].

Bolukbas y cols. ${ }^{11}$, concluyeron que el tratamiento preoperatorio con broncodilatadores de acción larga: Long-Acting Muscarinic Antagonist (LAMA) como tiotropio y Long-Acting Beta Agonist (LABA) como formoterol, y corticoides inhalatorios (budesonida) -en conjunto con cese del hábito tabáquico y kinesioterapia respiratoria- durante la semana previa a la cirugía de pacientes con diagnóstico reciente de EPOC y que serían sometidos a cirugía por cáncer pulmonar, mejora significativamente su función pulmonar preoperatoria: aumento del $\mathrm{VEF}_{1}$ (Volumen Espiratorio Forzado al $1^{\text {er }}$ segundo) y disminución de la gravedad del EPOC, además de disminuir las complicaciones postoperatorias como neumonía, retención de mucus y requerimiento de fibrobroncoscopias seriadas. También se obtienen estos mismos beneficios, aunque en menor magnitud, al tratar los pacientes solamente con broncodilatadores de acción larga (tiotropio y formoterol) por una semana.

En otro estudio retrospectivo, Kobayashi y cols. $^{12}$, analizaron 36 pacientes con EPOC y cáncer pulmonar que fueron sometidos a lobectomías y neumonectomías. Veintiún pacientes recibieron tiotropio inhalatorio y 4 de ellos (con $\mathrm{VEF}_{1} \%<60 \%$ ) inhalaciones con salmeterol las 2 semanas previas al procedimiento. Además, 8 de los pacientes dejaron de fumar las 2 semanas previas a la cirugía. Todos los pacientes significativamente mejoraron su función pulmonar y redujeron la severidad del EPOC, aumentando su capacidad vital funcional y $\mathrm{VEF}_{1}$.

El 2007, Starobin y cols. ${ }^{13}$, publicaron un estudio prospectivo y randomizado, de 90 pacientes que serían sometidos a cirugía de revascularización miocárdica, en el cual incluyeron 30 pacientes con EPOC que recibieron 2 a 3 semanas previas a la cirugía betametasona intramuscular $(2 \mathrm{mg}$ de liberación rápida y $5 \mathrm{mg}$ de liberación lenta), 30 pacientes con EPOC que recibieron una inyección de suero fisiológico como placebo y 30 pacientes sin EPOC como controles. El grupo tratado con corticoides presentó significativamente menos días de hospitalizaciones en Unidad de Cuidados Intensivos (UCI) y en régimen de baja complejidad (sala) que el grupo placebo.

Bingol y cols. ${ }^{14}$, en un estudio randomizado de 40 pacientes con EPOC y que serían sometidos a cirugía de revascularización miocárdica, administraron $20 \mathrm{mg}$ diarios de prednisolona oral a 20 pacientes y placebo oral a los otros 20, durante los 10 días preoperatorios y hasta el día del alta médica. Ambos grupos no presentaban diferencias significativas en sus cifras de $\mathrm{VEF}_{1}$ antes de recibir estos tratamien- tos. Los resultados mostraron que el grupo que recibió prednisolona mejoró significativamente el $\mathrm{VEF}_{1}$ de la espirometría preoperatoria $(63,2 \pm 4,24 \%$ vs $57,9 \pm 4,38 \%)$ y al alta médica $(55,15 \pm 2,98 \%$ vs $47,05 \pm 3,22 \%$ ) respecto del grupo control. Además, el grupo control presentó significativos mayores tiempos medios de extubación, tasas de reintubación y días de hospitalización en sala y en UCI.

Un estudio retrospectivo publicado por Savas y cols. ${ }^{15}$, el 2013, comparó 53 pacientes con EPOC que fueron sometidos a cirugía de revascularización miocárdica y que recibieron broncodilatadores y corticoides inhalatorios 10 días previos a la cirugía, con 51 pacientes que no recibieron ningún tipo de tratamiento. Los resultados mostraron que el grupo que recibió broncodilatadores y corticoides presentó significativos menores tiempos de extubación y menor incidencia de derrames pleurales, fibrilaciones auriculares y necesidad de soporte inotrópico, además de menores estadías hospitalarias en sala y en UCI.

\section{Tabaquismo}

En los últimos años, el tabaquismo ha aumentado significativamente en el mundo, convirtiéndose en un problema de salud pública en todo el orbe. En Chile, un 40,6\% de los pacientes fuman activamente $^{3}$, por lo que podemos extrapolar que un importante porcentaje de población que será sometida a cirugía tendrá asociado tabaquismo activo. El antecedente de este hábito es un factor de riesgo independiente para el desarrollo de complicaciones respiratorias postoperatorias, las cuales son la mayor causa de muerte después de una resección pulmonar, llegando hasta un $84 \%{ }^{16}$. La escala de Melbourne es un indicador que permite objetivar la presencia o ausencia de una complicación pulmonar postoperatoria después de una cirugía pulmonar (Tabla 1$)^{16}$. Con el fin de disminuir significativamente estas complicaciones, se ha propuesto la suspensión del hábito tabáquico preoperatorio.

Una revisión sistemática de 13 estudios, publicada el 2014 por Thomsen y cols. ${ }^{17}$, concluyó en 2 estudios que los pacientes en los cuales se realizaron intervenciones preoperatorias mayores para la suspensión del tabaquismo, correspondientes a consejerías semanales para dejar de fumar -presenciales o telefónicas- durante un período entre 4 y 8 semanas previas a la cirugía, tuvieron un RR de 0,42 (IC 0,27-0,65) para desarrollar cualquier tipo de complicación postoperatoria, siendo esta disminución significativa respecto a los controles. Otros 4 estudios con intervenciones menores, correspondientes a una única consejería dentro de las 4 semanas previas a la cirugía, tuvieron un $R R$ de 
0,92 (IC 0,72-1,19), no siendo esta reducción significativa con respecto a los controles. Además, 6 de los estudios con intervenciones menores y los 2 estudios con intervenciones mayores, ofrecieron terapia de reemplazo con nicotina. Ninguno de los 13 estudios encontró diferencias significativas en la aparición de complicaciones cardíacas y/o pulmonares postoperatorias comparando ambas intervenciones.

Mills y cols. ${ }^{18}$, publicaron una revisión sistemática y meta-análisis el 2011, el cual incluyó 6 estudios randomizados y 15 observacionales, demostrando que en los 6 estudios randomizados hubo una reducción significativa [RR 0,59 (IC $0,41-0,85$ )] de complicaciones postoperatorias en aquellos pacientes que suspendieron el tabaco en el período preoperatorio. Cada semana de cese del tabaquismo aumentaba la magnitud del efecto positivo en $19 \%$. Intervenciones en las cuales se dejó el tabaco menos de 4 semanas antes de la cirugía no tuvieron una reducción significativa del riesgo [RR 0,92 (IC 0,53$1,60)]$. En cambio, las intervenciones en las cuales se dejó el tabaco por más de 4 semanas tuvieron una disminución del riesgo de complicaciones postoperatorias estadísticamente significativa [RR 0,45 (IC $0,30-0,68)]$. Los estudios observacionales también encontraron importantes efectos en la suspensión del tabaquismo, disminuyendo el número total de complicaciones postoperatorias [RR 0,76 (IC 0,69-0,84)]. Esto también se observó para reducir significativamente las complicaciones de herida operatoria [RR 0,73 (IC, 0,61-0,87)] y pulmonares [RR 0,81 (IC 0,70-0,93)]. Finalmente, los estudios observacionales demostraron que períodos más largos de abstinencia de tabaco (más de 4 semanas), comparados con períodos más cortos (menos de 4 semanas), presentaban un promedio de reducción de complicaciones de un $20 \%$ [RR 0,80 (IC 0,67$0,97)]$.

Tabla 1. Escala de Melbourne $\geq \mathbf{4}$ de los 8 factores indica la presencia de complicaciones pulmonares postoperatorias $^{13}$

Variables
1. Radiografía de tórax con atelectasia o infiltrado
2. Esputo purulento
3. Diagnóstico clínico de neumonía
4. Temperatura corporal $>38^{\circ} \mathrm{C}$
5. Saturación de $\mathrm{O}_{2}<$ ambiental
6. Cultivo de expectoración bronquial positivo
7. Glóbulos blancos $>11.200$ unidades
8. Reingreso hospitalario o estadía prolongada en UCI

Otra revisión sistemática y meta-análisis realizada por Wong y cols. ${ }^{19}$, el 2011, incluyó 25 estudios (16 retrospectivos, 7 de cohorte y 2 randomizados). En comparación con los fumadores actuales, el riesgo de complicaciones respiratorias postoperatorias era similar en personas que dejaban de fumar menos de 2 semanas antes de la cirugía [RR 1,20 (IC, 0,961,50)] y entre 2 y 4 semanas [RR 1,14 (IC 0,90 $1,45)]$. Los fumadores que dejaban el tabaco más de 4 o más de 8 semanas previas a la cirugía, presentaban menores riesgos de complicaciones respiratorias postoperatorias que los fumadores actuales [RR 0,77 (IC 0,61-0,96)] y [RR 0,53 (IC 0,37-0,76)], respectivamente. Para las complicaciones secundarias a cicatrización de herida operatoria, el riesgo era menor en personas que dejaban el tabaco 3 a 4 semanas previas a la cirugía, en comparación con los fumadores actuales [RR 0,69 (IC 0,56-0,84)]. Cabe destacar también que los pacientes que continuaban fumando al momento de la cirugía, presentaron un aumento significativo de complicaciones pulmonares postoperatorias, comparados con los no fumadores [RR 2,11 (IC 1,51-2,94)].

Myers y cols. ${ }^{20}$, publicaron una revisión sistemática y meta-análisis el 2011, la cual incluyó 9 estudios. Sólo 1 encontró un efecto benéfico de la abstinencia reciente al tabaco (dentro de las 8 semanas previas a la cirugía), comparado con los fumadores actuales y ninguno encontró un efecto perjudicial. En el meta-análisis, dejar de fumar dentro de las 8 semanas previas a la cirugía no se asoció con aumento o disminución de las complicaciones postoperatorias totales, para todos los estudios disponibles [RR 0,78 (IC 0,57-1,07)]. Tampoco se observó un aumento o una disminución significativa de las complicaciones pulmonares postoperatorias [RR 1,18 $(0,95-1,6)]$.

\section{Asma}

La iniciativa GINA (Global Iniciative for Asth$m a$ ) define el asma como "una enfermedad heterogénea, normalmente caracterizada por una inflamación crónica de la vía aérea, con historia de síntomas respiratorios como sibilancias, disnea, opresión torácica y tos que varían a lo largo del tiempo y en intensidad, junto con una limitación espiratoria del flujo aéreo, la cual es variable"21.

La prevalencia del asma infantil en Chile fluctúa entre $7,3 \%$ y $16,5 \%{ }^{22,23}$ produciendo deterioro de la calidad de vida relacionada con salud ${ }^{24}$. Se desconoce la prevalencia de adultos asmáticos en Chile, sin embargo, según la Encuesta Nacional de Salud de 2009-2010, un 24,5\% de los chilenos presenta síntomas respiratorios crónicos ${ }^{2}$, de los cuales un porcentaje se atribuye al asma, por lo que muy probablemente nos encontremos con población 
quirúrgica que padezca esta enfermedad, haciéndose indispensable un buen manejo perioperatorio de estos pacientes.

Dones y cols. ${ }^{25}$, plantean que la hiperreactividad bronquial basal no controlada en niños asmáticos es el factor de riesgo más importante para presentar complicaciones pulmonares postoperatorias. Una adecuada historia clínica preoperatoria, puede detectar a pacientes con un inadecuado control de su asma o con una infección respiratoria actual o reciente, en quienes se deberían suspender las cirugías electivas. Los $\beta 2$ agonistas inhalatorios (salbutamol) preoperatorios parecieran ser útiles previniendo el aumento de la resistencia de la vía aérea después de la intubación traqueal en niños con asma leve a moderada ${ }^{22}$.

Scalfaro y cols. ${ }^{26}$, realizaron el 2001 un estudio randomizado y doble ciego, en el cual se le dio salbutamol o placebo vía inhalatoria, 30 a $60 \mathrm{~min}$ previos a la cirugía, a niños con asma leve a moderada que serían sometidos a cirugía electiva. Posterior a la intubación, los niños tratados con salbutamol mostraron una disminución significativa de la resistencia de vía aérea [6\% (IC $-25,2 \%$ a $+13,2 \%$ )], mientras que los tratados con placebo, tuvieron un aumento [17,7\% (IC 4,4\% a 30,9\%)].

En un estudio randomizado, doble ciego y prospectivo, Silvanus y cols. ${ }^{27}$, estudiaron 31 pacientes con obstrucción parcialmente reversible de la vía aérea y quienes nunca habían recibido tratamiento respiratorio, siendo asignados aleatoriamente a recibir diariamente por 5 días, vía inhalatoria, salbutamol solo $(\mathrm{n}=16)$ o salbutamol inhalatorio combinado con metilprednisolona oral $(n=15)$. Desde el primer día de tratamiento, tanto el grupo salbutamol solo como el grupo salbutamol-metilprednisolona disminuyeron significativamente la resistencia de la vía aérea y aumentaron significativamente su $\mathrm{VEF}_{1}$ y capacidad vital, sin diferencia entre ambos grupos. Por otro lado, sólo 1 paciente del grupo salbutamolmetilprednisolona experimentó sibilancias post extubación traqueal, a diferencia del grupo salbutamol solo, donde la mayoría de los pacientes sí presentó sibilancias, existiendo diferencias significativas entre ambos grupos.

En 1995, Kabalin y cols. ${ }^{28}$, hicieron un estudio retrospectivo que evaluó a 71 pacientes asmáticos, leves a severos, sometidos a 89 procedimientos quirúrgicos. Se determinó cualquier tipo de complicación postoperatoria y su incidencia, después de ser tratados con corticoides orales preoperatoriamente. Los pacientes tratados con corticoides no mostraron un aumento significativo del número de infecciones con respecto al grupo control, concluyendo que el tratamiento con corticoides preoperatorios no aumenta significativamente las complicaciones infecciosas postoperatorias.
Applegate y cols. ${ }^{29}$, el 2013 realizaron una revisión, sugiriendo que las alternativas terapéuticas previas a la cirugía de los pacientes con asma se basan en el grado de severidad de su enfermedad. Los asmáticos bien controlados pueden sólo necesitar $\beta-2$ agonistas inhalatorios el mismo día de la cirugía, mientras que los asmáticos moderados podrían requerir adición de corticoides inhalatorios a los $\beta 2$ agonistas descritos una semana antes de la cirugía. Finalmente, los asmáticos severos o mal controlados podrían necesitar agregar corticoides orales a los $\beta 2$ agonistas y corticoides inhalatorios antes señalados.

En otra revisión realizada por Tirumalasetty y cols. ${ }^{30}$, el 2006, plantean que si el paciente asmático no presenta síntomas previos a la cirugía o si no ha tenido crisis en el último año, no requerirían de ningún tratamiento preoperatorio. Sin embargo, si el paciente es usuario de broncodilatadores inhalatorios y no tiene historia de uso de corticoides orales, debería ser tratado con corticoides inhalatorios, como beclometasona o equivalente, una semana previa a la cirugía. Si el paciente ya está siendo tratado con corticoides inhalatorios, debe indicársele prednisona oral $0,5 \mathrm{mg}^{*} \mathrm{~kg}^{-1}$ los 5 días previos a la cirugía. Si el paciente es usuario crónico de corticoides orales, entonces la dosis debe ser incrementada, probablemente al doble los 5 días previos a la cirugía. Además, los pacientes que ya están usando corticoides inhalatorios u orales crónicamente, deberían también recibir hidrocortisona $100 \mathrm{mg}$ intravenosa cada $8 \mathrm{~h}$, empezando la mañana previa a la cirugía y continuándola postoperatoriamente hasta estabilizar los síntomas respiratorios.

\section{Conclusiones}

Las enfermedades respiratorias son la $3^{\mathrm{a}}$ causa de muerte en Chile y se ha visto un aumento importante de las patologías respiratorias crónicas en el mundo -como EPOC, tabaquismo y asma- por lo cual nos enfrentaremos a un número creciente de pacientes con estas enfermedades que serán sometidos en algún momento a procedimientos quirúrgicos. Se requiere de un adecuado manejo perioperatorio de estos pacientes con el fin de evitar complicaciones respiratorias postoperatorias.

De acuerdo a la evidencia médica actualmente disponible, es posible recomendar que los pacientes con EPOC y que serán sometidos a cirugía de cáncer pulmonar, deben recibir un tratamiento la semana previa al procedimiento con broncodilatadores inhalatorios de acción larga: LAMA como tiotropio y LABA como formoterol, junto con corticoides inhalatorios (budesonida). En los pacientes con EPOC y que serán sometidos a cirugía de revascularización miocárdica, recomendamos el uso de corticoides 
sistémicos preoperatorios, orales o intramusculares. Estas intervenciones mejoran significativamente la función pulmonar preoperatoria y disminuyen las complicaciones pulmonares postoperatorias.

Los pacientes fumadores deben suspender el cigarrillo por lo menos 4 semanas antes de la cirugía, con lo cual disminuirán significativamente sus complicaciones postoperatorias, especialmente las respiratorias y las relacionadas con la cicatrización de la herida operatoria. Debe realizarse una intervención intensiva a los pacientes para que dejen de fumar, incluyendo consejerías presenciales semanales y terapia de reemplazo con nicotina. No se ha demostrado que la suspensión del tabaco disminuya las complicaciones postoperatorias en pacientes fumadores sin patología respiratoria asociada.
En pacientes asmáticos, el tratamiento preoperatorio depende de la gravedad de su enfermedad y se recomienda el siguiente tratamiento preoperatorio:

- Asmáticos leves o bien controlados: $\beta 2$ agonistas inhalatorios el mismo día de la cirugía.

- Asmáticos moderados: $\beta 2$ agonistas + corticoides inhalatorios durante una semana previa a la cirugía.

- Asmáticos severos o mal controlados: $\beta 2$ agonistas + corticoides inhalatorios + corticoides orales durante una semana previa a la cirugía.

El resumen de las recomendaciones y los fármacos, vía de administración, dosis y duración del tratamiento preoperatorio en cada una de las patologías antes descritas se encuentran en la Tabla 2.

Tabla 2. Resumen de recomendaciones de tratamiento preoperatorio de pacientes con enfermedad pulmonar obstructiva crónica (EPOC), tabaquismo y asma bronquial

\begin{tabular}{|c|c|c|c|}
\hline Patología & Referencia & Recomendación & Dosis \\
\hline \multirow[t]{5}{*}{ EPOC } & Bolukbas $^{11}$ & $\begin{array}{l}\text { Dejar de fumar } \\
\text { Kinesioterapia respiratoria } \\
\text { Broncodilatadores de } \\
\text { acción larga } \\
\text { Corticoides inhalatorios }\end{array}$ & $\begin{array}{l}\text { Tiotropio puff } 18 \mathrm{mcg}^{*} \text { día }^{-1} \\
\text { Formoterol puff } 9 \text { mcg cada } 12 \mathrm{~h} \\
\text { Budesonida puff } 400 \text { mcg cada } 12 \mathrm{~h}\end{array}$ \\
\hline & $\begin{array}{l}\text { Kobayashi }^{12} \text { (Lobectomía, } \\
\text { Neumonectomía) }\end{array}$ & $\begin{array}{l}\text { Dejar de fumar } \\
\text { Broncodilatadores de } \\
\text { acción larga }\end{array}$ & $\begin{array}{l}\text { Tiotropio puff } 18 \mathrm{mcg}^{*} \text { día }^{-1} \\
\text { Salmeterol puff } 50 \mathrm{mcg} \text { cada } 12 \mathrm{~h}\end{array}$ \\
\hline & $\begin{array}{l}\text { Starobin }^{13} \text { (Revascularización } \\
\text { miocárdica) }\end{array}$ & Corticoides sistémicos & $\begin{array}{l}\text { Betametasona intramuscular } 5 \mathrm{mg} \text { de liberación } \\
\text { lenta y } 2 \mathrm{mg} \text { de liberación rápida }\end{array}$ \\
\hline & $\begin{array}{l}\text { Bingol }^{14} \text { (Revascularización } \\
\text { miocárdica) }\end{array}$ & Corticoides sistémicos & Prednisolona oral $20 \mathrm{mg}^{*}$ día $^{-1}$ por 20 días \\
\hline & $\begin{array}{l}\text { Savas }^{15} \text { (Revascularización } \\
\text { miocárdica) }\end{array}$ & $\begin{array}{l}\text { Broncodilatadores } \\
\text { Corticoides }\end{array}$ & $\begin{array}{l}\text { Según recomendación GOLD } \\
\text { Según recomendación GOLD }\end{array}$ \\
\hline \multirow[t]{2}{*}{ Tabaquismo } & Thomsen $^{17}$ & Dejar de fumar & $\begin{array}{l}\text { Consejería presencial o telefónica } \\
\text { Terapia de reemplazo de nicotina: } \\
\text { - vareniclina } 2 \mathrm{mg}^{*} \text { día }^{-1} \text { por } 1 \mathrm{mes} \\
\text { - parches nicotina } 1 \text { parche por día }(30 \mathrm{mg}) \\
\text { - chicles SOS hasta } 12 \text { chicles }(2 \mathrm{mg}) \text { al día }\end{array}$ \\
\hline & Mills $^{18}$, Wong $^{19}$, Myers $^{20}$ & Dejar de fumar & \\
\hline \multirow[t]{8}{*}{ Asma } & Dones $^{25}$ & Broncodilatadores & Salbutamol puff $400 \mathrm{mcg}$ SOS y antes de la cirugía \\
\hline & Scalfaro ${ }^{26}$ & Broncodilatadores & Salbutamol puff $400 \mathrm{mcg}$ antes de la cirugía \\
\hline & Silvanus $^{27}$ & Broncodilatadores & Salbutamol puff $200 \mathrm{mcg} \mathrm{c} / 8 \mathrm{~h}$ por 5 días \\
\hline & & Corticoides sistémicos & Metilprednisolona oral $40 \mathrm{mg}^{*}$ día $^{-1}$ por 5 días \\
\hline & Kabalin $^{28}$ & Broncodilatadores & Manejo habitual según GINA ${ }^{21}$ \\
\hline & & Corticoides sistémicos & $\begin{array}{l}\text { Prednisona oral } 1 \mathrm{mg}^{*} \mathrm{~kg}^{-1} * \mathrm{dí}^{-1} \text { por 3-7 días } \\
\text { (máximo } 60 \mathrm{mg} \text { diarios) }\end{array}$ \\
\hline & Applegate $^{29}$ & Broncodilatadores & Salbutamol puff $400 \mathrm{mcg}$ SOS \\
\hline & & $\begin{array}{l}\text { Corticoides sistémicos/ } \\
\text { inhalatorios }\end{array}$ & Según recomendación GINA ${ }^{21}$ \\
\hline
\end{tabular}


Ciertamente, aún se necesita mayor evidencia respecto al adecuado manejo preoperatorio de pacientes con enfermedades respiratorias crónicas para disminuir las complicaciones postoperatorias. Recomendamos siempre una evaluación multidisciplinaria previa a la cirugía de estos pacientes, involucrando al cirujano, anestesiólogo y médico internista o broncopulmonar tratante de su patología de base.

\section{Referencias}

1. Instituto Nacional de Estadísticas (INE). Anuarios de Estadísticas Vitales, Chile, 2011.

2. Ministerio de Salud Gobierno de Chile, Facultad de Medicina Pontificia Universidad Católica de Chile, Observatorio Social Universidad Alberto Hurtado. Encuesta nacional de salud 2009-2010 Tomo I, V. Prevalencias expandidas de problemas de salud.

3. Ministerio de Salud Gobierno de Chile, Facultad de Medicina Pontificia Universidad Católica de Chile, Observatorio Social Universidad Alberto Hurtado. Encuesta nacional de salud 2009-2010 Tomo II, V. Resultados, V.2.5. Exposición a tabaco.

4. Johnson RG, Arozullah AM, Neumayer L, Henderson WG, Hosokawa P, Khuri SF. Multivariable predictors of postoperative respiratory failure after general and vascular surgery: results from the patient safety in surgery study. J Am Coll Surg. [Comparative Study]. 2007;204:1188-98.

5. Vestbo J, Hurd SS, Agusti AG, Jones PW, Vogelmeier C, Anzueto A, et al. Global strategy for the diagnosis, management, and prevention of chronic obstructive pulmonary disease: GOLD executive summary. Am J Respir Crit Care Med. [Review]. 2013 15;187:347-65.

6. Menezes AM, Pérez-Padilla R, Jardim JR, Muino A, López MV, Valdivia G, et al. Chronic obstructive pulmonary disease in five Latin American cities (the PLATINO study): a prevalence study. Lancet 2005;366(9500):1875-81.

7. Leo F, Venissac N, Pop D, Solli P, Filosso P, Minniti A, et al. Postoperative exacerbation of chronic obstructive pulmonary disease. Does it exist? Eur J Cardiothorac Surg. [Multicenter Study]. 2008;33:424-9.

8. Smetana GW, Lawrence VA, Cornell JE, American College of P. Preoperative pulmonary risk stratification for noncardiothoracic surgery: systematic review for the American College of Physicians. Ann Intern Med. [Research Support, U.S. Gov't, Non-P.H.S. Review]. 2006;144:581-95.

9. Arozullah AM, Daley J, Henderson WG, Khuri SF. Multifactorial risk index for predicting postoperative respiratory failure in men after major noncardiac surgery. The National Veterans Administration Surgical Quality Improvement Program. Ann Surg. [Multicenter
Study Research Support, U.S. Gov't, Non-P.H.S.]. 2000;232:242-53.

10. Arozullah AM, Khuri SF, Henderson WG, Daley J, Participants in the National Veterans Affairs Surgical Quality Improvement P. Development and validation of a multifactorial risk index for predicting postoperative pneumonia after major noncardiac surgery. Ann Intern Med. [Research Support, U.S. Gov't, Non-P.H.S. Validation Studies]. 2001;135:847-57.

11. Bolukbas S, Eberlein M, Eckhoff J, Schirren J. Shortterm effects of inhalative tiotropium/formoterol/budenoside versus tiotropium/formoterol in patients with newly diagnosed chronic obstructive pulmonary disease requiring surgery for lung cancer: a prospective randomized trial. Eur J Cardiothorac Surg. [Comparative Study Randomized Controlled Trial]. 2011;39:995-1000.

12. Kobayashi S, Suzuki S, Niikawa H, Sugawara T, Yanai M. Preoperative use of inhaled tiotropium in lung cancer patients with untreated COPD. Respirology 2009;14:675-9.

13. Starobin D, Kramer MR, Garty M, Shitirt D. Morbidity associated with systemic corticosteroid preparation for coronary artery bypass grafting in patients with chronic obstructive pulmonary disease: a case control study. J Cardiothorac Surg. [Randomized Controlled Trial]. 2007;2:25.

14. Bingol H, Cingoz F, Balkan A, Kilic S, Bolcal C, Demirkilic U, et al. The effect of oral prednisolone with chronic obstructive pulmonary disease undergoing coronary artery bypass surgery. J Card Surg. [Clinical Trial Comparative Study Randomized Controlled Trial]. 2005;20:252-6.

15. Savas Oz B, Kaya E, Arslan G, Karabacak K, Cingoz F, Arslan M. Pre-treatment before coronary artery bypass surgery improves post-operative outcomes in moderate chronic obstructive pulmonary disease patients. Cardiovasc J Afr. [Comparative Study]. 2013;24:184-7.

16. Agostini P, Cieslik H, Rathinam S, Bishay E, Kalkat MS, Rajesh PB, et al. Postoperative pulmonary complications following thoracic surgery: are there any modifiable risk factors? Thorax 2010;65:815-8.

17. Thomsen T, Villebro N, Moller AM. Interventions for preoperative smoking cessation. Cochrane Database Syst Rev. 2014;3:CD002294.

18. Mills E, Eyawo O, Lockhart I, Kelly S, Wu P, Ebbert JO. Smoking cessation reduces postoperative complications: a systematic review and meta-analysis. Am J Med. [Meta-Analysis Research Support, Non-U.S. Gov't Review]. 2011;124:144-54 e8.

19. Wong J, Lam DP, Abrishami A, Chan MT, Chung F. Short-term preoperative smoking cessation and postoperative complications: a systematic review and metaanalysis. Can J Anaesth. [Meta-Analysis Research Support, Non-U.S. Gov’t Review]. 2012;59:268-79.

20. Myers K, Hajek P, Hinds C, McRobbie H. Stopping smoking shortly before surgery and postoperative 
complications: a systematic review and meta-analysis. Arch Intern Med. [Meta-Analysis Research Support, Non-U.S. Gov't Review]. 2011;171:983-9.

21. Bateman ED, Hurd SS, Barnes PJ, Bousquet J, Drazen JM, Fitz Gerald M, et al. Global strategy for asthma management and prevention: GINA executive summary. Eur Respir J. 2008;31:143-78.

22. Mallol J, Aguirre V, Aguilar P, Calvo M, Amarales L, Arellano P y cols. Cambios en la prevalencia de asma en escolares chilenos entre 1994 y 2002: International Study of Asthma and Allergies in Childhood (ISAAC)Chile phases I and III. Rev Med Chile 2007;135:580-6.

23. Mallol VJ, Cortez QE, Amarales OL, Sánchez DI, Calvo GM, Soto LS y cols. Prevalencia del asma en escolares chilenos: Estudio descriptivo de 24.470 niños. ISAACChile. Rev Med Chile 2000;128:279-85.

24. Velástegui C, Pérez-Canto $P$, Zárate V, Arenas D, Salinas P, Moreno G y cols. Impacto del asma en escolares de dos centros de salud primaria. Rev Med Chile 2010;138:205-12.

25. Dones F, Foresta G, Russotto V. Update on perioperative management of the child with asthma. Pediatr Rep. 2012;4:e19.
26. Scalfaro P, Sly PD, Sims C, Habre W. Salbutamol prevents the increase of respiratory resistance caused by tracheal intubation during sevoflurane anesthesia in asthmatic children. Anesth Analg. [Clinical Trial Randomized Controlled Trial Research Support, Non-U.S. Gov't]. 2001;93:898-902.

27. Silvanus MT, Groeben H, Peters J. Corticosteroids and inhaled salbutamol in patients with reversible airway obstruction markedly decrease the incidence of bronchospasm after tracheal intubation. Anesthesiology. [Clinical Trial Comparative Study. Randomized Controlled Trial]. 2004;100:1052-7.

28. Kabalin CS, Yarnold PR, Grammer LC. Low complication rate of corticosteroid-treated asthmatics undergoing surgical procedures. Arch Intern Med. [Research Support, Non-U.S. Gov’t]. 1995;155:137984.

29. Applegate R, Lauer R, Lenart J, Gatling J, Vadi M. The Perioperative Management of Asthma. J Aller Ther S. 2013;11:2.

30. Tirumalasetty J, Grammer LC. Asthma, surgery, and general anesthesia: a review. J Asthma. [Comparative Study Review]. 2006;43:251-4. 